\title{
Evaluation of interrelations between resistin, adiponectin, PAI-1, intercellular adhesion molecule and thyroid function in autoimmune thyroid disease
}

Celestino Neves ${ }^{1}$, João Sérgio Neves ${ }^{1}$, Sofia Castro Oliveira ${ }^{1}$, César Esteves ${ }^{1}$, Oksana Sokhatska ${ }^{2}$,

Luís Delgado ${ }^{2}$, José Luís Medina ${ }^{1} \&$ Davide Carvalho ${ }^{1,3}$

${ }^{1}$ Department of Endocrinology, Diabetes and Metabolism, São João Hospital Center; Faculty of Medicine of the University of Porto (FMUP);

${ }^{2}$ Immunology Department, São João Hospital, FMUP; ${ }^{3}$ Instituto de Investigação e Inovação em Saúde, University of Porto, Porto, Portugal.

\section{Introduction}

Thyroid function has a major effect on body weight, energy metabolism and adipocytes physiology.

\section{Methods}

We evaluated 98 patients with autoimmune thyroid disease divided into three groups: 30 who were euthyroid (TSH=0.35-4.94 Ul/ml), 35 with subclinical hypothyroidism (hypoSC, TSH >4.94 UI/ml) and 33 with subclinical hyperthyroidism (hiperSC, TSH $<0.35 \mathrm{UI} / \mathrm{ml}$ ). We evaluated BMI, serum concentration of FT3, FT4, TSH, C-reactive protein (CRP), adiponectin, resistin, PAI1 (plasminogen activator inhibitor-1) and ICAM-1. The statistical analysis was performed using ANOVA, Student's t-test and Spearman's correlations.

\section{Objective}

Our aim was to study the impact of subclinical thyroid dysfunction in plasmatic levels of adipokines and ICAM-1.

Results

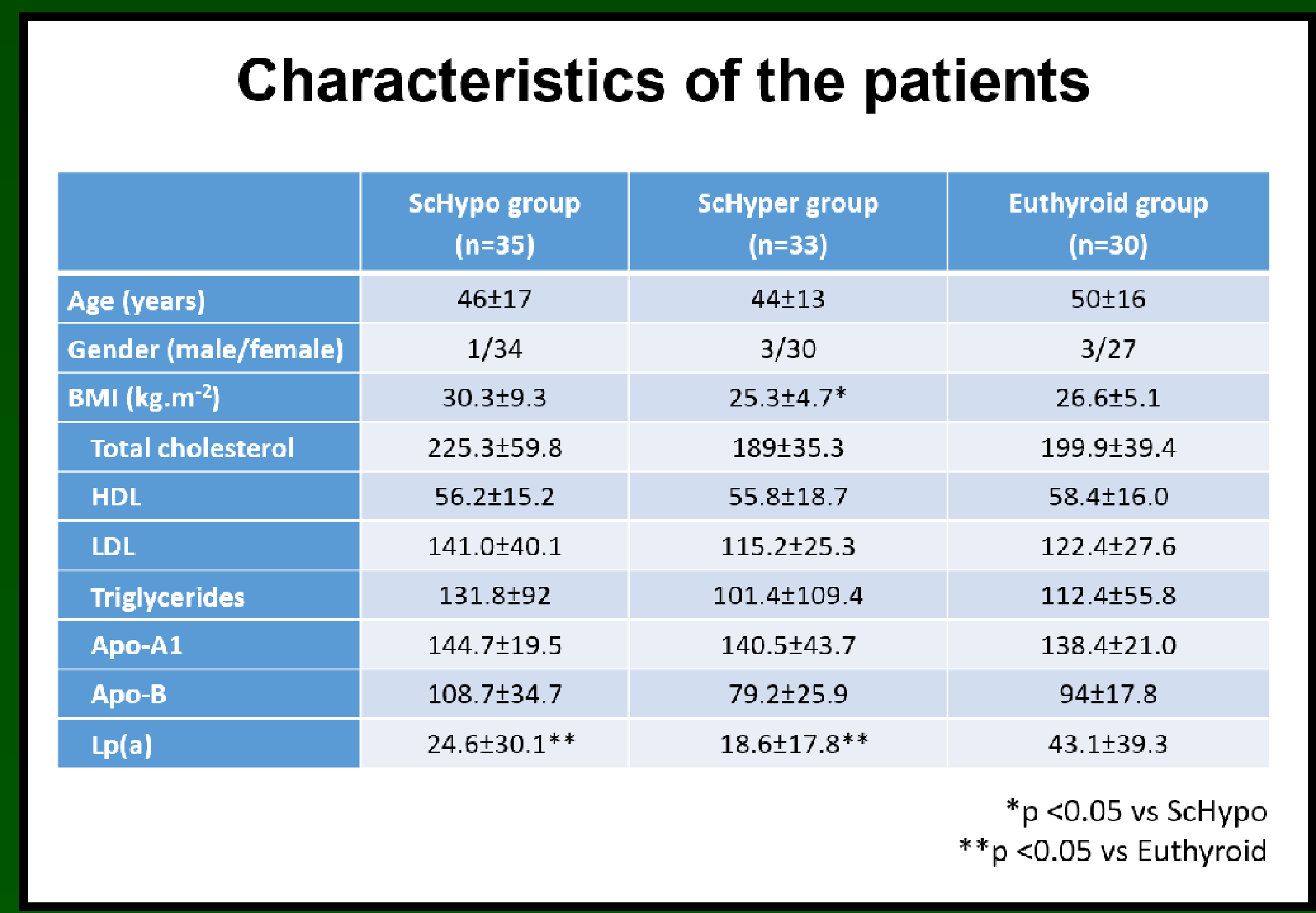

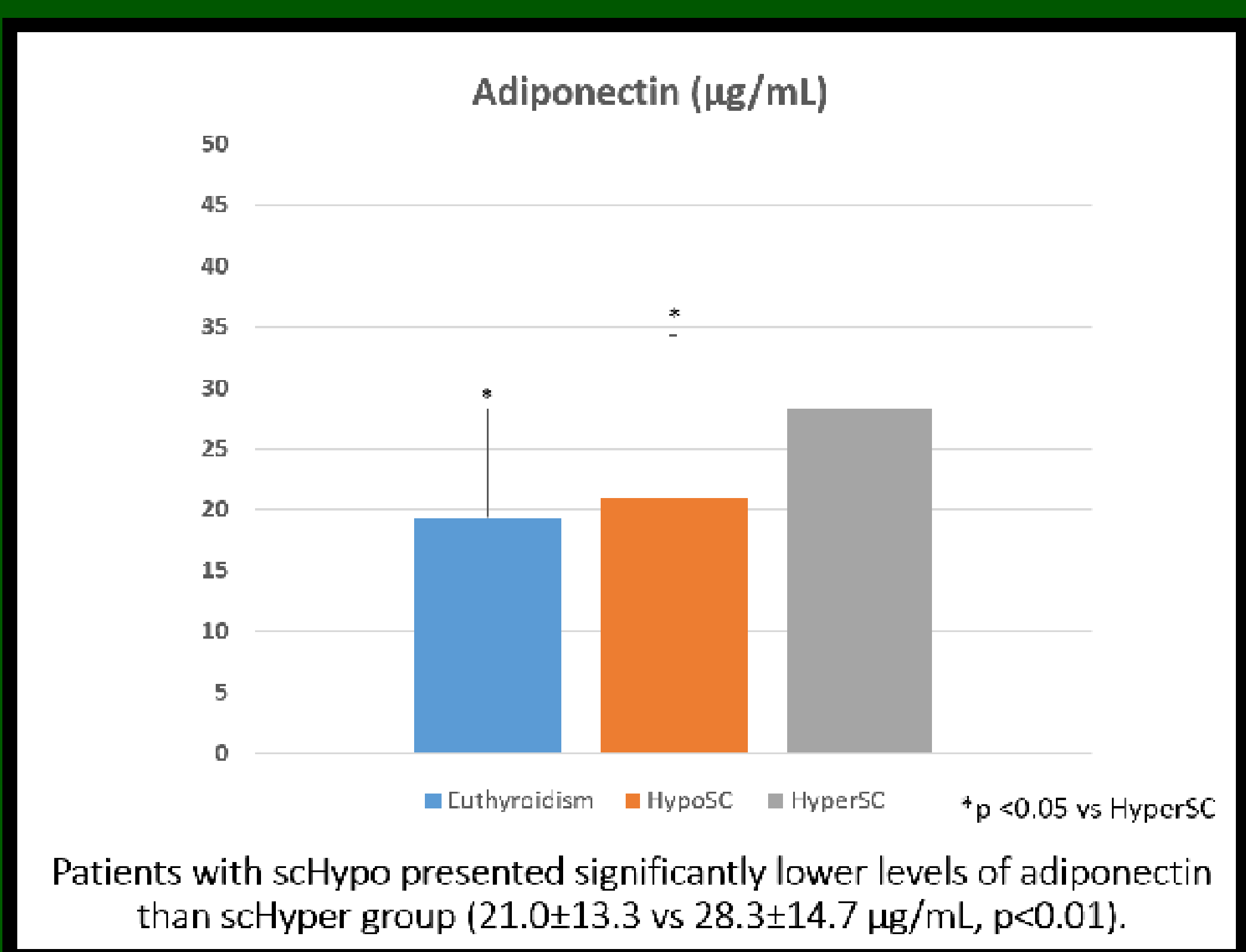
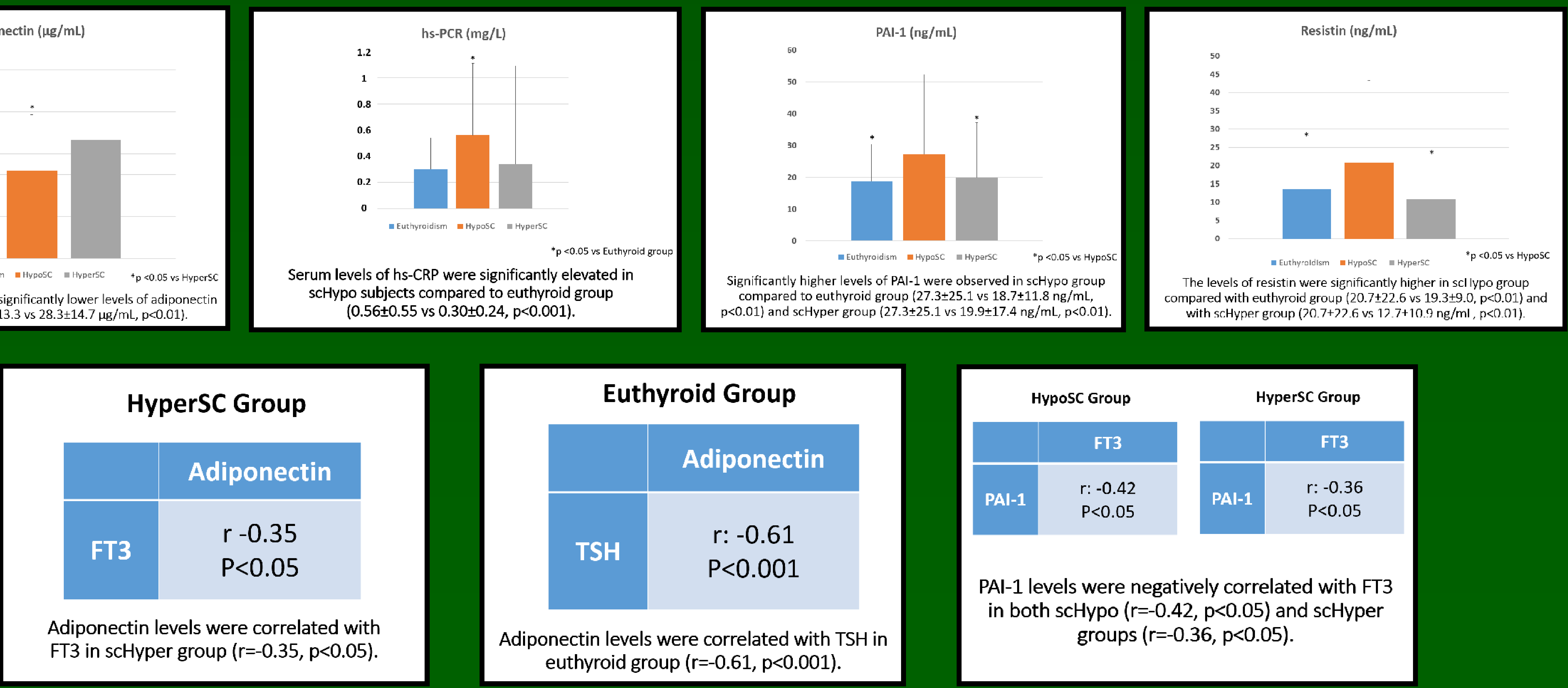

\section{Conclusions}

We found significantly higher levels of resistin and PAI-1 in subclinical hypothyroidism. The interrelations between thyroid function, adipokines and ICAM-1 may contribute to the metabolic and cardiovascular complications in autoimmune thyroid disease. 